Article

\title{
Computational Analysis of Exotic Molecular and Atomic Vibrations in Ice XV
}

\author{
Xiao-Ling Qin $₫$, Xu-Liang Zhu ${ }^{\circledR}$, Jing-Wen Cao, Lu Jiang, Yue Gu, Xue-Chun Wang and \\ Peng Zhang * (1)
}

School of Space Science and Physics, Shandong University, Weihai 264209, China

* Correspondence: zhangpeng@sdu.edu.cn; Tel.: +86-631-568-8751

Received: 19 August 2019; Accepted: 26 August 2019; Published: 27 August 2019

check for updates

\begin{abstract}
It is always difficult to assign the peaks of a vibrational spectrum in the far-infrared region. The two distinct peaks seen in many ice phases are still a mystery to date. The normal modes of ice XV were calculated using the CASTEP code based on first-principles density functional theory. On the basis of vibrational modes analysis, we divided the translational modes into three categories: four-bond vibrations, which have the highest energy levels; two-bond vibrations, which have medium levels of energy; and relative vibrations between two sublattices, which have the lowest energy. Whale et al. found that some intramolecular stretching modes include the isolated vibration of only one $\mathrm{O}-\mathrm{H}$ bond, whereas the others do not vibrate in ice $\mathrm{XV}$. We verified this phenomenon in this study and attributed it to local tetrahedral deformation. Analysis of normal modes, especially in the translation and stretching band of ice $\mathrm{XV}$, clarified the physical insights of the vibrational spectrum and can be used with other ice phases.
\end{abstract}

Keywords: ice XV; translational modes; hydrogen bond; CASTEP; density functional theory

\section{Introduction}

More than 17 ice phases have been experimentally identified at various temperatures and pressures [1]. These phases can be divided into two main categories on the basis of hydrogen long-range order or disorder. Among ice phases, seven hydrogen-ordered phases are currently known, ices II, VIII, IX, XI, XII, XIV, and XV. Most of them can be converted into hydrogen-disordered ice phase, in which the water molecules exhibit orientation disorder after heating. There are six known counterparts: Ih/XI, III/IX, V/XIII, VI/XV, VII/VIII, and XII/XIV [2]. The only exception is ice II, for which a hydrogen-disordered counterpart has not been observed. Upon heating, it transforms to either ice Ill or ice V depending on the pressure. The high-pressure phase of ice VI was first discovered by Bridgman [3]. The crystal structure of ice VI was solved by Kamb via X-ray diffraction at ambient pressure at the temperature of liquid nitrogen [4], and confirmed by Kuhs et al. via neutron diffraction [5,6]. Considering only oxygen lattices, the primitive cell of ice VI contains 10 water molecules with space group $\mathrm{P} 4_{2} / \mathrm{nmc}$, which is a hydrogen-disordered phase. Ice VI consists of two interpenetrating yet independent hydrogen-bonded networks, and has thus been described as a "self-clathrate" [4].

In 2009, Salzmann et al. found a new hydrogen-ordered phase by doping $\mathrm{HCl}$ in ice VI at a lower temperature, which is called ice XV. This hydrogen-ordered structure is antiferroelectric $(P \overline{1})$ and can be stable from $0.8 \mathrm{GPa}$ to $1.5 \mathrm{GPa}$ at temperatures below $130 \mathrm{~K}$ [7]. Whale et al. measured the Raman spectrum and performed simulations with the first-principles density functional theory (DFT) [8]. Salzmann et al. later presented another hydrogen-ordered structure of VI, the ferromagnetic structure with space group Cc $[9,10]$. In 2018, Gasser et al. demonstrated the existence of one more XV phase, which they called the ice beta-XV phase [11]. 
For theoretical investigations of vibrational modes, the most interesting results were those of Whale et al., who found exotic "rigid network" molecular vibrations in the translation band and "isolated stretching" modes of atomic vibrations in the stretching band [8]. We also found "rigid network" modes in ice VIII and VII [2] and "isolated stretching" modes in ice XIV [12]. According to our series of investigations of ice phases, we identified two intrinsic categories of molecule vibrations in the translational band that constitute two sharp peaks [2,12-15]. In this paper, we confirm the presence of cluster vibrations in a much lower region of the translation band. We review the normal modes in ice XV and aim to clarify the physical insights of these exotic vibration modes.

\section{Computational Methods}

We first constructed the oxygen structure of ice VI. Then, we used a self-compiled program to find two possible hydrogen-ordered structures. We selected the antiferromagnetic one that is the same as reported by Whale for this work. The density is $1.32 \mathrm{~g} / \mathrm{cm}^{3}$, in which the three orthorhombic constants of a primitive cell are $\mathrm{a}=6.23 \AA, \mathrm{b}=6.22 \AA$, and $\mathrm{c}=5.83 \AA$. Using the CASTEP [16] code based on first-principles density functional theory (DFT), we calculated the phonon density of states (PDOS) of antiferroelectric ice XV. The energy threshold and self-consistent field tolerance were set as $1 \times$ $10^{-9} \mathrm{eV} /$ atom. The energy cutoff was $750 \mathrm{eV}$, and the k-point was $2 \times 2 \times 2$. As the electron density has a large distribution in ice lattice, we chose generalized gradient approximation (GGA) for this work. To test the exchange-correlation (XC) functional, we tried RPBE, PBE, PW91, WC, and PBESOL separately for geometric optimization. The results showed that the densities are 1.21, 1.40, 1.41, 1.55, and $1.56 \mathrm{~g} / \mathrm{cm}^{3}$, respectively. As the length of intermolecular hydrogen bond (H-bond) is relative to volume change and the vibrational frequency is very sensitive to $\mathrm{H}$-bond length, the best selection is RPBE [17], which produces a slight redshift in the translation band, but greater accuracy in the intramolecular vibrations. The hydrostatic pressure was set to 0.9 and $1.4 \mathrm{GPa}$ for comparison with the $\mathrm{k}$ mesh was set as $4 \times 4 \times 4$. We found the H-bond frequencies of $1.4 \mathrm{GPa}$ have a big redshift than $0.9 \mathrm{GPa}$ (please see Supplementary File S1). Thus, we selected the simulation data of $0.9 \mathrm{GPa}$ for discussions hereinafter. The optimized dimensions are $\mathrm{a}=6.39 \AA \mathrm{A}, \mathrm{b}=6.44 \AA$, and $\mathrm{c}=6.02 \AA$.

\section{Results and Discussion}

Figure 1 shows the computing spectra of Raman scattering, infrared (IR) absorption, and PDOS, which are divided into four parts according to four separate vibrational regions. Because of the wide scale of the intensity in different regions, we adjusted their proportions for comparison. A primitive cell contains 10 molecules; thus, there are $10 \times 3 \times 3-3=87$ optical normal modes.

Table 1 shows the details of PDOS, normal modes, and Raman scattering peaks. Column 1 lists the main peaks of PDOS. We identified 34 distinguishable peaks, of which 11 are major. The PDOS curve integrates phonon signals throughout the Brillouin zone (BZ), so it could correspond to an inelastic neutron scattering (INS) spectrum. Because reports of INS data are lacking, we present PDOS herein for reference. The 87 normal modes are shown in column 2, and the IR and Raman active intensities are shown in the next two columns. Sixteen Raman peaks are measured in the experiment: 114, 158, 186, 220, 230, 300, 312, 387, 451, 499, 548, 3224, 3335, 3345, 3396, and $3425 \mathrm{~cm}^{-1}$ [8]. However, the peak at $387 \mathrm{~cm}^{-1}$ did not match any normal mode. We doubt that this is a false peak because a gap should be present between the translation and libration bands. There is a slight underestimation of the hydrogen bond (H-bond) by RPBE, but it is more accurate than Whale's work by PBE [8].

In the translational band, the Raman peaks are from optic modes near the BZ that are detectable subject to selection rules. On the basis of harmonic approximation, 27 optic normal modes are present between 73 and $285 \mathrm{~cm}^{-1}$. Note that the peaks are matched with normal modes by the calculated intensities. 

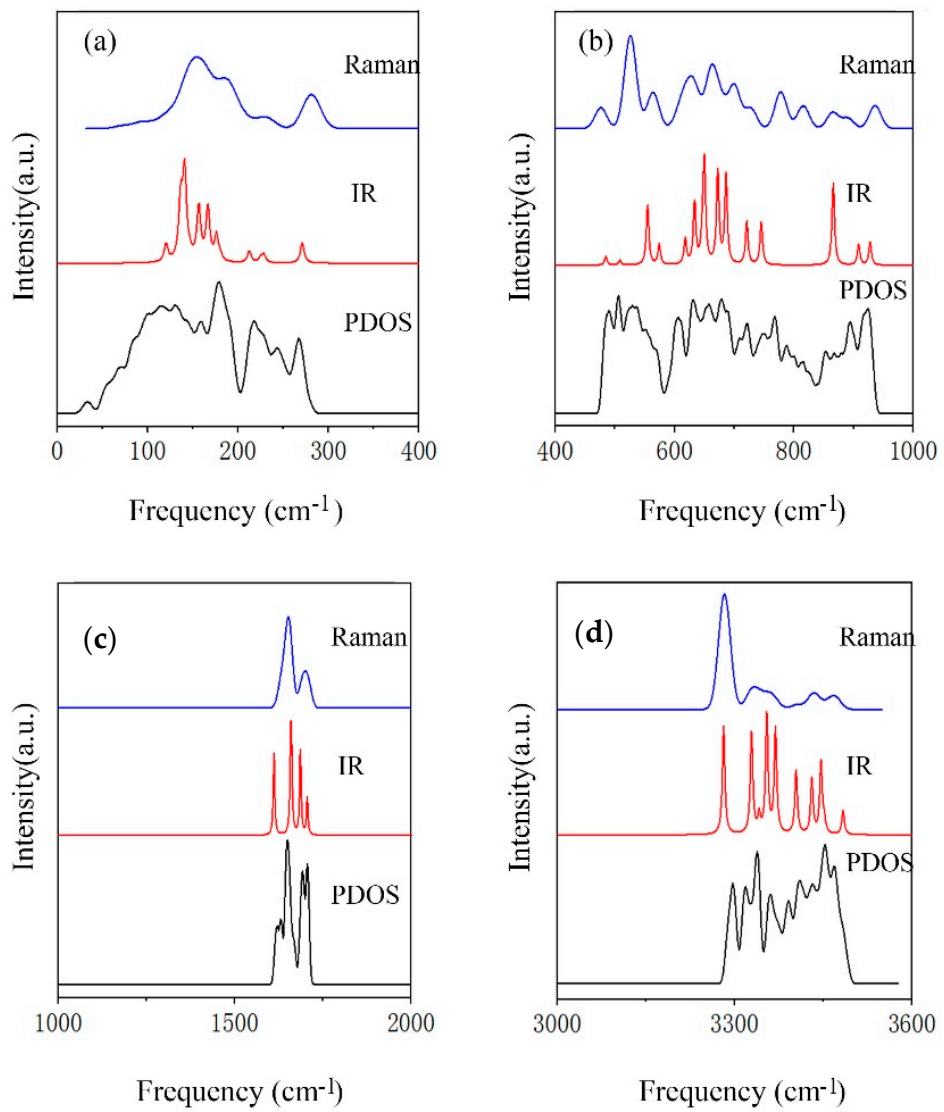

Figure 1. Computational spectra of Raman, IR, and phonon density of states (PDOS) of ice XV. Four vibrational bands: (a) intermolecular translation band; (b) intermolecular libration band; (c) intramolecular bending band; and (d) intramolecular stretching band.

Table 1. Comparison of calculated results with Raman data. The normal modes (0.9 GPa) with IR/Raman intensities are compared with experimental Raman peaks. N means Raman inactive. PDOS, phonon density of states.

\begin{tabular}{ccccc}
\hline PDOS & Normal Modes & IR Intensity & Raman Intensity & Raman Exp. [8/11] \\
\hline \multirow{6}{*}{$\mathbf{1 1 3}$} & 73 & $\mathrm{~N}$ & 0.12 & \\
& 106 & $\mathrm{~N}$ & 0.24 & \\
& 107 & $\mathrm{~N}$ & 0.15 & \\
$\mathbf{1 3 0}$ & 136 & $\mathrm{~N}$ & 0.22 & $114 / 113$ \\
& 138 & 1.65 & $\mathrm{~N}$ & \\
& 148 & $\mathrm{~N}$ & 0.72 & \\
& 150 & 1.14 & $\mathrm{~N}$ & \\
& 158 & 1.60 & $\mathrm{~N}$ & \\
& 162 & $\mathrm{~N}$ & 0.27 & \\
& 165 & $\mathrm{~N}$ & 1.20 & \\
$\mathbf{1 5 9}$ & 167 & 1.39 & $\mathrm{~N}$ & \\
& 175 & 0.92 & $\mathrm{~N}$ & \\
& 181 & 0.24 & $\mathrm{~N}$ & \\
$\mathbf{1 7 8}$ & 182 & $\mathrm{~N}$ & 0.57 & \\
& 184 & $\mathrm{~N}$ & 0.40 & \\
& 185 & 1.22 & $\mathrm{~N}$ & \\
& 192 & $\mathrm{~N}$ & 1.55 & \\
& 201 & 0.22 & $\mathrm{~N}$ & \\
& 206 & $\mathrm{~N}$ & 0.33 & \\
& 221 & $\mathrm{~N}$ & 0.32 & $220 / 215$ \\
\hline
\end{tabular}


Table 1. Cont.

\begin{tabular}{|c|c|c|c|c|}
\hline PDOS & Normal Modes & IR Intensity & Raman Intensity & Raman Exp. [8/11] \\
\hline \multirow[t]{4}{*}{217} & 225 & 0.37 & $\mathrm{~N}$ & \\
\hline & 231 & 0.33 & $\mathrm{~N}$ & \\
\hline & 232 & $\mathrm{~N}$ & 0.04 & $230 /$ \\
\hline & 234 & 0.13 & $\mathrm{~N}$ & \\
\hline 243 & 236 & $\mathrm{~N}$ & 0.30 & $300 /$ \\
\hline \multirow[t]{3}{*}{268} & 275 & 0.64 & $\mathrm{~N}$ & \\
\hline & 285 & $\mathrm{~N}$ & 1.01 & $312 / 303$ \\
\hline & 477 & $\mathrm{~N}$ & 1.81 & $451 / 450$ \\
\hline 489 & 484 & 1.09 & $\mathrm{~N}$ & \\
\hline 506 & 508 & 0.80 & $\mathrm{~N}$ & \\
\hline \multirow[t]{4}{*}{529} & 531 & $\mathrm{~N}$ & 7.56 & $499 / 495$ \\
\hline & 550 & 9.21 & $\mathrm{~N}$ & \\
\hline & 566 & $\mathrm{~N}$ & 3.06 & $548 / 544$ \\
\hline & 579 & 2.93 & $\mathrm{~N}$ & \\
\hline \multirow[t]{3}{*}{606} & 611 & $\mathrm{~N}$ & 1.43 & \\
\hline & 618 & 4.52 & $\mathrm{~N}$ & \\
\hline & 621 & $\mathrm{~N}$ & 2.42 & \\
\hline \multirow[t]{4}{*}{631} & 631 & 10.29 & $\mathrm{~N}$ & \\
\hline & 635 & $\mathrm{~N}$ & 3.31 & \\
\hline & 649 & 1.36 & $\mathrm{~N}$ & \\
\hline & 652 & 16.64 & $\mathrm{~N}$ & \\
\hline \multirow[t]{2}{*}{657} & 662 & $\mathrm{~N}$ & 4.75 & \\
\hline & 671 & 15.25 & $\mathrm{~N}$ & \\
\hline \multirow[t]{3}{*}{679} & 677 & $\mathrm{~N}$ & 1.89 & \\
\hline & 685 & 8.19 & $\mathrm{~N}$ & \\
\hline & 702 & $\mathrm{~N}$ & 3.94 & \\
\hline \multirow[t]{2}{*}{722} & 718 & 5.70 & $\mathrm{~N}$ & \\
\hline & 731 & $\mathrm{~N}$ & 1.79 & \\
\hline 747 & 748 & 5.20 & $\mathrm{~N}$ & \\
\hline 768 & 775 & $\mathrm{~N}$ & 3.73 & \\
\hline \multirow[t]{3}{*}{815} & 817 & $\mathrm{~N}$ & 2.33 & \\
\hline & 864 & 11.87 & $\mathrm{~N}$ & \\
\hline & 867 & $\mathrm{~N}$ & 1.51 & \\
\hline \multirow[t]{2}{*}{894} & 892 & $\mathrm{~N}$ & 1.08 & \\
\hline & 913 & 2.73 & $\mathrm{~N}$ & \\
\hline \multirow[t]{2}{*}{926} & 928 & 3.25 & $\mathrm{~N}$ & \\
\hline & 936 & $\mathrm{~N}$ & 1.97 & \\
\hline 1621 & 1609 & 5.37 & $\mathrm{~N}$ & \\
\hline 1630 & 1629 & $\mathrm{~N}$ & 1.94 & \\
\hline \multirow[t]{5}{*}{1649} & 1648 & $\mathrm{~N}$ & 3.68 & \\
\hline & 1655 & $\mathrm{~N}$ & 5.27 & \\
\hline & 1656 & 5.46 & $\mathrm{~N}$ & \\
\hline & 1660 & 3.70 & $\mathrm{~N}$ & \\
\hline & 1684 & 5.57 & $\mathrm{~N}$ & \\
\hline \multirow[t]{2}{*}{1692} & 1692 & $\mathrm{~N}$ & 2.71 & \\
\hline & 1705 & 2.37 & $\mathrm{~N}$ & \\
\hline \multirow[t]{2}{*}{1706} & 1709 & $\mathrm{~N}$ & 2.91 & \\
\hline & 3278 & 63.85 & $\mathrm{~N}$ & \\
\hline \multirow[t]{2}{*}{3296} & 3280 & $\mathrm{~N}$ & 3777.67 & $3224 / 3222$ \\
\hline & 3325 & 57.33 & $\mathrm{~N}$ & \\
\hline 3318 & 3326 & $\mathrm{~N}$ & 552.40 & $3335 /$ \\
\hline \multirow[t]{3}{*}{3338} & 3339 & 7.64 & $\mathrm{~N}$ & \\
\hline & 3341 & $\mathrm{~N}$ & 346.17 & $3345 / 3349$ \\
\hline & 3354 & 57.53 & $\mathrm{~N}$ & \\
\hline
\end{tabular}


Table 1. Cont.

\begin{tabular}{ccccc}
\hline PDOS & Normal Modes & IR Intensity & Raman Intensity & Raman Exp. [8/11] \\
\hline 3360 & 3357 & $\mathrm{~N}$ & 288.67 & \\
& 3366 & $\mathrm{~N}$ & 278.88 & \\
$\mathbf{3 3 9 1}$ & 3367 & 50.96 & $\mathrm{~N}$ & \\
$\mathbf{3 4 1 1}$ & 3402 & 27.87 & $\mathrm{~N}$ & $3396 /$ \\
& 3403 & $\mathrm{~N}$ & 164.02 & $3425 / 3420$ \\
$\mathbf{3 4 3 2}$ & 3429 & 27.91 & $\mathrm{~N}$ & \\
& 3432 & $\mathrm{~N}$ & 394.53 & \\
3453 & 3442 & $\mathrm{~N}$ & 154.45 & \\
& 3448 & 39.83 & $\mathrm{~N}$ & \\
$\mathbf{3 4 6 9}$ & 3452 & 9.24 & $\mathrm{~N}$ & \\
& 3461 & $\mathrm{~N}$ & 100.59 & \\
& 3472 & $\mathrm{~N}$ & 383.12 & \\
\hline
\end{tabular}

Our previous study of ice Ic revealed two kinds of intrinsic translational modes in the ice lattice [13]. In the strong mode, the molecule vibrates together with four connecting H-bonds, which is called the four-bond mode. Two perpendicular degenerate weak modes involve only two oscillating H-bonds, which is called the two-bond mode. Treating the two H-bonded molecules as spring, a simple harmonic oscillator model yielded a strength ratio of $\sqrt{2}$.

Although the tetrahedral structure of ice $\mathrm{XV}$ presents deformation under pressure to some extent, these two kinds of modes can also be distinguished in the translation region. For the mode at $285 \mathrm{~cm}^{-1}$ shown in Figure 2, typical molecules vibrate along its $\mathrm{HOH}$ angular bisector, which yields a strong vibrational mode (please see Supplementary File S2). Note that some small vibrations of other molecules can keep the mass center of a primitive cell static. The mode at $206 \mathrm{~cm}^{-1}$ is a two-bond mode, in which a molecule vibrates toward its two neighbors (please see Supplementary File S3). Whale et al. found that the three lowest modes at 66,78 , and $85 \mathrm{~cm}^{-1}$ present vibrations of the two rigid ice $X V$ networks. In this study, the three modes are at 73, 106, and $107 \mathrm{~cm}^{-1}$, respectively. They actually represent three orthorhombic vibrations in 3D space that originate from the interactions of two sublattices without $\mathrm{H}$-bonds, and thus present the lowest vibrational energy. Figure 2 illustrates an example of the mode at $107 \mathrm{~cm}^{-1}$ (please see the dynamic process from Supplementary File S4). We also found this phenomenon in ice VII and VIII [1]; however, the most common case in other ice phases is that many cluster vibration or skeleton deformation modes are present in the much lower region. Considering the translational band of ice, we can divide normal modes into three categories: four-bond vibrations, which possess the highest energy; two-bond vibrations, with medium energy levels; and cluster vibrations, which have the lowest energy. Figure 3 shows a diagram of fitted curves of three kinds of modes in ice XV compared with the inserted PDOS. Note that the data for normal modes are in the BZ center, whereas the real PDOS covers the entire first BZ. Thus, some disagreements occur as a result of the dispersion of phonons.

As shown in Figure 2, the libration band contains 30 normal modes from 477 to $936 \mathrm{~cm}^{-1}$. There are three types of vibration modes: rocking, or rotation of the whole molecule around an axis perpendicular to the molecular plane; twisting, or rotation around an axis coincident with the $\mathrm{HOH}$ angle bisector; and wagging, or rotation of the molecule around an axis in the molecular plane, perpendicular to the bisector of the $\mathrm{HOH}$ angle. For an optic mode, the mass center of the primitive cell remains static while the molecules within vibrate. Figure 2 shows that the main vibrating mode at $477 \mathrm{~cm}^{-1}$ is rocking, whereas the mode at $913 \mathrm{~cm}^{-1}$ is twisting. For the highest frequency at $936 \mathrm{~cm}^{-1}$, the vibrating mode of molecules is a mixture of wagging, rocking, and twisting.

The bending band has 10 normal modes from 1609 to $1709 \mathrm{~cm}^{-1}$, as shown in Figure 4. The vibration pattern of each molecule is bending, whereas the collective vibrations can be distinguished as in-phase and out-of-phase. For the highest-frequency mode at $1709 \mathrm{~cm}^{-1}$, the vibrating mode of crystal lattice is 
in-phase. The lowest mode at $1609 \mathrm{~cm}^{-1}$ is out-of-phase. The mode at $1684 \mathrm{~cm}^{-1}$ shown in Figure 4 has the greatest intensity of IR absorption, but has no Raman activity. In contrast, the mode at $1655 \mathrm{~cm}^{-1}$ has the greatest intensity of Raman activity, but no IR activity in this band. Because the intensities are very small compared with the stretching band, it is always difficult to detect signals for IR and Raman experiments.

There are 20 normal modes in the stretching band from 3278 to $3485 \mathrm{~cm}^{-1}$, including intramolecular symmetrical and asymmetrical stretching vibrations. For the lowest frequency at $3281 \mathrm{~cm}^{-1}$, all molecules show symmetric stretching. For the frequency at $3461 \mathrm{~cm}^{-1}$, the main vibrations show asymmetric stretching. The mode at $3280 \mathrm{~cm}^{-1}$ has the greatest intensity of Raman activity, but no IR activity, whereas the opposite is found in the mode at $3278 \mathrm{~cm}^{-1}$.

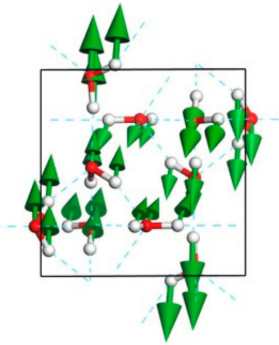

107

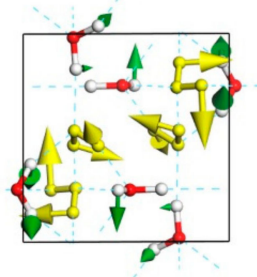

477

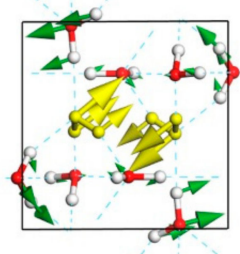

206

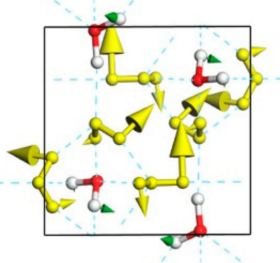

913

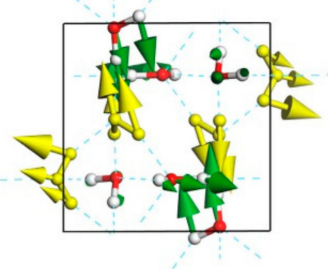

285

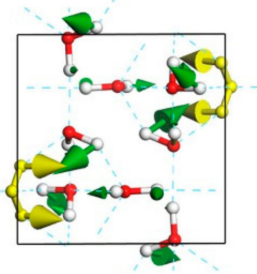

936

Figure 2. Top view of six normal modes in translational band $\left(107,206\right.$, and $\left.285 \mathrm{~cm}^{-1}\right)$ and libration band $\left(477,913\right.$, and $\left.936 \mathrm{~cm}^{-1}\right)$. Typical molecular vibrations are shown in gold.

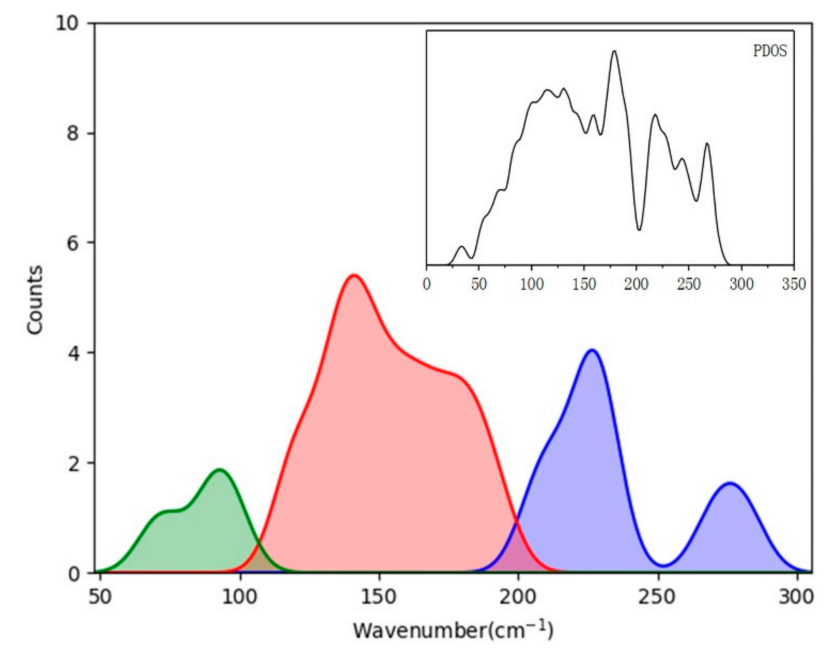

Figure 3. Fitted curves of four-bond modes (blue), two-bond modes (red), and non-H-bond modes (green) of ice XV in translational band. Inset PDOS curve is shown for comparison. 


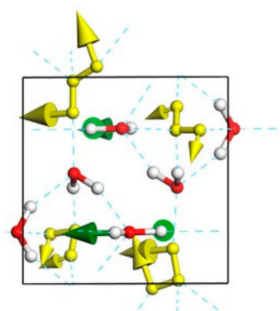

1609

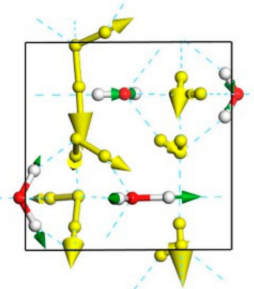

3278

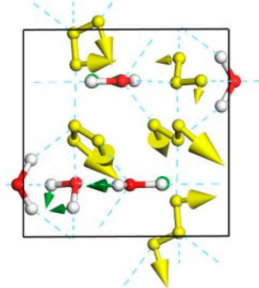

1656

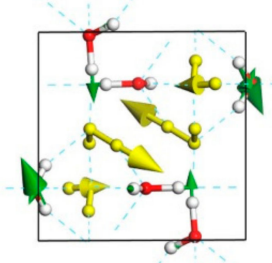

3366

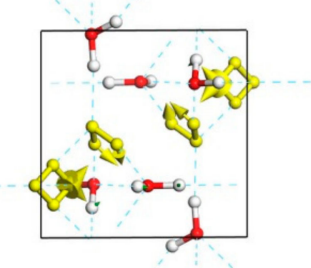

1709

Figure 4. Top view of six normal modes in bending band $\left(1609,1656\right.$, and $\left.1709 \mathrm{~cm}^{-1}\right)$ and stretching band $\left(3278,3366\right.$, and $\left.3461 \mathrm{~cm}^{-1}\right)$, respectively. Mode at $3366 \mathrm{~cm}^{-1}$ is a typical isolated stretching vibration.

Whale et al. reported the normal modes in this band; some vibrational modes include isolated vibration of only one $\mathrm{O}-\mathrm{H}$ bond, whereas the other does not vibrate [8]. A typical example is shown in Figure 4 of the mode at $3367 \mathrm{~cm}^{-1}$ (please see Supplementary File S5). We also found this vibration pattern in other ice phases, such as ice XIV and VII. We regard this "isolated stretching" mode as a special case of asymmetric stretching and attribute it to local tetrahedral deformation by pressure and a hydrogen-disordered lattice.

\section{Conclusions}

It is traditionally difficult to assign the peaks of a vibrational spectrum in the fingerprint region. We analyze the normal modes of ice XV via DFT simulation. On the basis of our series of investigations in the translational band of ice, we divide normal modes into three categories: four-bond vibrations that possess the highest energies; two-bond vibrations that possess medium energies; and cluster vibrations that have the lowest energy. In ice XV, VII, and VIII, the cluster modes are relative vibrations between two sublattices. Thus, we can analyze the translational modes in the far IR region of ice. This study presents physical insights into the vibrational spectrum and can be used with other ice phases.

In addition, we reviewed the "isolated stretching" mode first reported by Whale et al. As we also found this phenomenon in other ice phases, we do not regard this mode as a third type in addition to symmetric and asymmetric stretching, and instead attribute it to a special asymmetric stretching due to local tetrahedral deformation by pressure and a hydrogen-disordered lattice.

Supplementary Materials: The following are available online. File S1: The results of calculations at 1.4 GPa and $0.9 \mathrm{GPa}$, the $\mathrm{k}$ mesh was set as $4 \times 4 \times 4$. Video S2: Dynamic process of four-bond vibration at $285 \mathrm{~cm}^{-1}$. Video S3: Dynamic process of two-bond vibration at $206 \mathrm{~cm}^{-1}$. Video S4: Dynamic process of relative vibration between two sublattices at $107 \mathrm{~cm}^{-1}$. Video S5: Dynamic process of isolated stretching at $3366 \mathrm{~cm}^{-1}$.

Author Contributions: X.-L.Q. drafted the manuscript. X.-L.Z. and J.-W.C. assisted structural modeling and data processing. L.J., Y.G., and X.-C.W. participated in the discussion of results. All authors gave final approval for publication. P.Z. conducted simulations and data analysis and edited the manuscript.

Funding: This research received no external funding.

Acknowledgments: The numerical calculations were conducted on the supercomputing system in the Supercomputing Center, Shandong University, Weihai.

Conflicts of Interest: The authors declare no conflicts of interest. 


\section{References}

1. Gu, Y.; Zhu, X.L.; Jiang, L. Comparative analysis of hydrogen bond vibrations in ice VIII and VII. J. Phys. Chem. C 2019, 123, 14880-14883. [CrossRef]

2. Moberg, D.R.; Sharp, P.J.; Paesani, F. Molecular-level interpretation of vibrational spectra of ordered ice phases. J. Phys. Chem. B 2018, 122, 10572-10581. [CrossRef] [PubMed]

3. Bridgman, P.W. Water, in the Liquid and Five Solid Forms, under Pressure. Proc. Am. Acad. Arts Sci. 1912, 47, 441. [CrossRef]

4. Kamb, B. Structure of ice VI. Science 1965, 150, 205. [CrossRef] [PubMed]

5. Kuhs, W.F.; Finney, J.L.; Vettier, C.; Bliss, D.V. Structure and hydrogen ordering in ices VI, VII, and VIII by neutron powder diffraction. J. Chem. Phys. 1984, 81, 3612. [CrossRef]

6. Kuhs, W.F.; Ahsbahs, H.; Londono, D.; Finney, J.L. In-situ crystal growth and neutron four-circle diffractometry under high pressure. Phys. B 1989, 684, 156-157. [CrossRef]

7. Salzmann, C.G.; Radaelli, P.G.; Mayer, E.; Finney, J.L. Ice XV: A new thermodynamically stable phase of ice. Phys. Rev. Lett. 2009, 103, 105701. [CrossRef] [PubMed]

8. Whale, T.F.; Clark, S.J.; Finney, J.L. DFT-assisted interpretation of the Raman spectra of hydrogen-ordered ice XV. J. Raman Spectrosc. 2013, 44, 290-298. [CrossRef]

9. Komatsu, K.; Noritake, F.; Machida, S. Partially ordered state of ice XV. Sci. Rep. 2016, 6, 28920. [CrossRef] [PubMed]

10. Nanda, K.D.; Beran, G.J.O. What governs the proton ordering in ice XV? J. Phys. Chem. Lett. 2013, 4, 3165-3169. [CrossRef]

11. Gasser, T.M.; Thoeny, A.; Plaga, L. Experimental evidence for a second hydrogen ordered phase of ice VI. Chem. Sci. 2018, 9, 4224-4234. [CrossRef] [PubMed]

12. Zhang, K.; Zhang, P.; Wang, Z.R.; Zhu, X.L.; Lu, Y.B.; Guan, C.B.; Li, Y. DFT simulations of the vibrational spectrum and hydrogen bonds of ice XIV. Molecules 2018, 23, 1781. [CrossRef] [PubMed]

13. Yuan, Z.Y.; Zhang, P.; Yao, S.K.; Lu, Y.B.; Yang, H.Z.; Luo, H.W.; Zhao, Z.J. Computational assignments of lattice vibrations of ice Ic. RSC Adv. 2017, 7, 36801. [CrossRef]

14. Wang, Z.R.; Zhu, X.L.; Jiang, L.; Zhang, K.; Luo, H.W.; Gu, Y.; Zhang, P. Investigations of the hydrogen bonds and vibrational spectra of clathrate ice XVI. Materials 2019, 12, 246. [CrossRef]

15. Zhu, X.L.; Yuan, Z.Y.; Jiang, L.; Zhang, K.; Wang, Z.R.; Luo, H.W.; Gu, Y.; Cao, J.W.; Qin, X.L.; Zhang, P. Computational analysis of vibrational spectrum and hydrogen bonds of ice XVII. New J. Phys. 2019, $21,043054$. [CrossRef]

16. Clark, S.J.; Segall, M.D.; Pickard, C.J.; Hasnip, P.J.; Probert, M.I.J.; Refson, K.; Payne, M.C. First principles methods using CASTEP. Z. Kristallogr. 2005, 220, 567-570. [CrossRef]

17. Hammer, B.; Hansen, L.B.; Norskov, J.K. Improved adsorption energetics within density-functional theory using revised Perdew-Burke-Ernzerhof functionals. Phys. Rev. B. 1999, 59, 7413-7421. [CrossRef] 